\title{
QUASIKONFORME FORTSETZBARKEIT, FREDHOLMSCHE EIGENWERTE UND GRUNSKYSCHE KOEFFIZIENTENBEDINGUNGEN
}

\author{
REINER KÜHNAU
}

\section{Einleitung}

Die folgenden Bemerkungen sollen im Anschluß an [10] den Zusammenhang zwischen den konformen Abbildungen mit einer quasikonformen Fortsetzung, den Fredholmschen Eigenwerten sowie den Grunskyschen Koeffizientenbedingungen behandeln. Dabei werden u.a. auch Eigenwertabschätzungen, z. T1. die genauen Eigenwerte für einige Kurven gewonnen.

Jeder geschlossenen Jordankurve $\mathbb{2}$ läßt sich bekanntlich (vgl. z. B. [18], S. 135, [17] und dortige Literatur) durch

$$
1 / \lambda=\sup \left|D(h)-D^{*}(h)\right| /\left[D(h)+D^{*}(h)\right]
$$

ein „Fredholmscher Eigenwert" $\lambda$ zuordnen. Dabei durchläuft $h$ alle durchweg stetigen Funktionen, die außerhalb und innerhalb von $\mathbb{L}$ jeweils harmonisch sind, und $D(h)$ bzw. $D^{*}(h)$ bezeichnet das Dirichletsche Integral von $h$ bezüglich des Äußeren bzw. Inneren von $\mathfrak{Q}$. Es gilt $\lambda>1$ genau für "Quasikreise”. Bei hinreichend glatten Kurven läßt sich bekanntlich $\lambda$ auch als Eigenwert bei einer klassischen Integralgleichung deuten.

Wie üblich sei nun $\Sigma$ die Klasse der schlichten konformen Abbildungen

$$
w(z)=z+\frac{a_{1}}{z}+\ldots
$$

von $|z|=1$. Wir entwickeln jeweils

$$
-\log \frac{w(z)-w(\zeta)}{z-\zeta}=\sum_{k, l=1}^{\infty} a_{k l} z^{-k \zeta-1}
$$

und nennen zu festem $w(z) \in \Sigma$ den kleinsten Faktor $x \leqq 1$, für den stets

$$
\left|\sum_{k, l=1}^{n} a_{k l} x_{k} x_{l}\right| \equiv \sum_{k=1}^{n}\left|x_{k}\right|^{2} / k
$$

mit beliebigen komplexen $x_{k}$ gültig ist, die ,Grunsky-Norm”" von $w(z)$.

Wir definieren nun drei Teilklassen von $\Sigma$ (vgl. auch [11], S. 287 ff.). 
Definition. Es sei für $0 \leqq x<1$

a) $\Sigma(x)$ die Gesamtheit aller $w(z) \in \Sigma$, bei denen eine $Q$-quasikonforme Fortsetzung nach $|z|<1$ möglich ist mit $\chi=(Q-1) /(Q+1)$,

b) $\Sigma\langle x\rangle$ die Gesamtheit aller $w(z) \in \Sigma$, bei denen $|z|=1$ in eine Jordankurve übergeht, deren Fredholmscher Eigenwert $\geqq 1 / \varkappa$ ist,

c) $\Sigma[\chi]$ die Gesamtheit aller $w(z) \in \Sigma$, bei denen die Grunsky-Norm $\leqq \varkappa$ ist.

In allen drei Fällen folgt $w(z) \equiv z$ bei $\varkappa=0$ (im Falle b) nach [15]). Nach [1] gilt $\Sigma(x) \leqq \Sigma\langle x\rangle$, nach [10] dabei $\Sigma(x) \neq \Sigma\langle x\rangle$. Nach [10] (vgl, dort Ende von S. 127) gilt weiter $\Sigma\langle x\rangle \subseteq \Sigma[\varkappa]$ (die entsprechende Aussage bei glatter Bildberandung auch in [13]). Unten werden wir schärfer $\Sigma\langle x\rangle=\Sigma[\varkappa]$ beweisen, was sozusagen eine ,,innere Definition" der Fredholmschen Eigenwerte ermöglicht. $\Sigma(x) \subseteq \Sigma[x]$ war schon in [4] bewiesen worden (vgl. u.a. auch [11]); daselbst auch weitere notwendige Bedingungen für Zugehörigkeit zu $\Sigma(\varkappa)$ bzw. $\Sigma[\varkappa]$, hinreichende solche sind zusammengestellt in [2]. Im folgenden sollen aus Bedingungen für Zugehörigkeit zu $\Sigma[\varkappa]$ noch Eigenwertabschätzungen gewonnen werden. In einigen Fällen ergeben sich sogar die genauen Eigenwerte. Dies ist wohl der Mitteilung nicht unwert, da bisher die Eigenwerte nur für wenige Kurven bekannt sind.

\section{Bedingungen für $\Sigma[\varkappa]$ und Eigenwertabschätzungen}

Da in [4] bei einigen für $\Sigma(\varkappa)$ angegebenen Ungleichungen tatsächlich nur (4) benutzt wurde, können wir folgende Ungleichungen für $\Sigma[\chi]$ notieren, was dann allemal unter Benutzung der Invarianz von $\lambda$ bei linearer Transformation zu Eigenwertabschätzungen Anlaß gibt.

a) Es gilt für $|z| \geqq 1$ bei $w(z) \in \Sigma[\chi]$ nach [4], (25) die (unscharfe) Ungleichung (das Bild von $|z|=1$ ist nach [11], S. 288 eine Jordankurve)

$$
|w(z)-z| \leqq c(\varkappa) /|z| \text { mit } \quad c(\varkappa)=\int_{1}^{\infty}\left[\left(1-r^{-2}\right)^{-\varkappa}-1\right] d r .
$$

Wegen

$$
\int x^{-2}\left(1-x^{2}\right)^{-x} d x=-x^{-1}\left(1-x^{2}\right)^{1-x}-(1-2 x) \int\left(1-x^{2}\right)^{-*} d x
$$

rechnet man noch leicht mit der Beta- und Gammafunktion um

$$
\begin{aligned}
c(x) & =\int_{0}^{1}\left[\left(1-x^{2}\right)^{-x}-1\right] x^{-2} d x=1-(1-2 \varkappa) \int_{0}^{1}\left(1-x^{2}\right)^{-\varkappa} d x \\
& =1-\left(\frac{1}{2}-\varkappa\right) B(1 / 2,1-\varkappa)=1-\sqrt{\pi} \frac{\Gamma(1-\varkappa)}{\Gamma\left(\frac{1}{2}-\varkappa\right)} .
\end{aligned}
$$


Jedenfalls ist $c(x)$ für $x=0$ analytisch mit $c(0)=0, c^{\prime}(0)=2 \log 2=1,386 \ldots$ Damit haben wir in $\Sigma[x]$ mit für $x \rightarrow 0$ beschränkten $O\left(x^{2}\right) / x^{2}$

$$
|w(z)-z| \leqq\left[1,387 x+O\left(x^{2}\right)\right] /|z| \text {. }
$$

Zum Vergleich: Es gilt nach [5] in $\Sigma(x)$

$$
|w(z)-z| \leqq\left[1,274 \varkappa+O\left(\varkappa^{2}\right)\right] /|z| .
$$

Aus (5), (6) folgt insbesondere für den Durchmesser $D$ des Bildes von $|z|=1$ bei $w(z) \in \Sigma[\varkappa]$

$$
(2 \leqq) D \leqq 2+2 c(\varkappa)=2+(4 \log 2) \varkappa+O\left(\varkappa^{2}\right) .
$$

Denn dieses Bild von $|z|=1 \mathrm{mu} ß$ in

$$
1-c(x) \leqq|w| \leqq 1+c(x)
$$

liegen, wobei der linke Teil der Ungleichung für $x \geqq 1 / 2$ (entsprechend $c(x) \geqq 1$ ) hinfällig wird. Dies liefert dann noch den

Satz 1. Wenn die geschlossene Jordankurve den Eigenwert $\lambda>2$ besitzt, dann ist sie enthalten in einem konzentrischen Kreisring mit dem Radienverhältnis $[1+c(1 / \lambda)] /[1-c(1 / \lambda)]$.

Anders herum gelesen: Läßt sich die Jordankurve nicht in einen konzentrischen Kreisring mit dem Radienverhältnis $[1+c(\varkappa)] /[1-c(\varkappa)]$ einbetten, dann gilt $\lambda \leqq 1 / \varkappa$. Diese Eigenwertabschätzung bringt natürlich nur etwas für kreisnahe Jordankurven.

b) Durch eine bekannte Substitution fließt noch aus (5) für $w(z) \in \Sigma[\varkappa]$

$$
\left|w^{\prime \prime}(z) / w^{\prime}(z)\right| \leqq 2 c(x) /\left[|z|\left(|z|^{2}-1\right)\right] \text { bei }|z|>1 \text {, }
$$

ähnlich wie z. B. (4) in [6], welche Ungleichung auch in $\Sigma[\chi]$ gilt.

c) Es gilt bei $|z|>1$ und $w(z) \in \Sigma[\varkappa]$ nach [4] (vgl. auch [11], S. 287) die scharfe Ungleichung

insbesondere

$$
\log w^{\prime}(z) \mid / \log \frac{1}{1-|z|^{-2}} \leqq \varkappa,
$$

$$
\left(1-|z|^{-2}\right)^{x} \leqq\left|w^{\prime}(z)\right| \leqq\left(1-|z|^{-2}\right)^{-\varkappa} .
$$

Sei nun das Bild von $|z|=1$ eine geschlossene Jordankurve, die in Umgebung eines ihrer Punkte aus zwei dort unter dem Winkel $\alpha \pi$ zusammenstoßenden analytischen Bögen besteht. Dann folgt nach (12) aus Kelloggschen Resultaten (vgl. z. B. [20], S. 365) $|1-\alpha| \leqq \chi$, falls $w(z) \in \Sigma[\varkappa]$. Dies bringt uns

Satz 2. Ist $\mathfrak{Q}$ eine geschlossene Jordankurve, die in Umgebung eines ihrer Punkte aus zwei dort unter dem Winkel $\alpha \pi \neq \pi$ zusammenstoßenden analytischen Bögen besteht, dann gilt für ihren Eigenwert

$$
\lambda \leqq 1 /|1-\alpha| .
$$


Falls 2 ein reguläres $n-E c k$ ist, liefert dies

$$
\lambda \leqq n / 2 \text {. }
$$

Nach [1], [17] gilt übrigens hierbei $\lambda \geqq 1 / \sin (\pi / n)$. Es erscheint lohnenswert, die Abschätzung $\lambda \geqq n / 2$ zu versuchen herzuleiten, indem eine quasikonforme Spiegelung konstruiert wird, bei der besondere Sorgfalt den Umgebungen der Ecken gewidmet wird. Überhaupt scheinen allgemein Ecken bei $\mathfrak{Q}$ einen erheblichen, i. allg. entscheidenden Einfluß auf $\mathfrak{Q}$ zu haben.

Dies erhellt auch durch folgendes Beispiel eines Kreisbogenzweieckes $\mathfrak{2}$ mit dem Winkel $\alpha \pi$. Durch lineare Transformation kann man o.E.d.A. annehmen, daß $\mathfrak{L}$ besteht aus der positiv reellen Achse und dem Strahl arg $z=\alpha \pi(0<\alpha<1)$ einer 3 -Ebene. Durch Affinität in der Ebene von $\log z$ findet man elementar eine $[(2 / \alpha)-1]-$ quasikonforme Spiegelung an $\mathcal{L}$, was nach [1] $\lambda \geqq 1|1-\alpha|$ nach sich zieht. Im Verein mit (13) haben wir damit

$$
i=1 /|1-\alpha|
$$

Ähnlich können wir den genauen Wert von $\lambda$ bestimmen, falls 2 die geschlossene ,Knickkurve" ist, die in der $z$-Ebene entsteht durch $3=\zeta^{\alpha} \quad(0<\alpha<2)$ aus dem Kreis $|\zeta-1|=1$ der $\zeta$-Ebene. Dabei nehmen wir den Zweig, der die rechte Halbebene $\mathfrak{R} \mathfrak{c} \zeta>0$ auf den Winkelraum $\mathfrak{W}$ abbildet, der durch $|\arg z|<\alpha \pi / 2$ definiert ist. Dadurch hat $\mathfrak{L}$ in $z=0$ ein Ecke des Winkels $\alpha \pi$ und ist für $0<\alpha<1$,,tropfenförmig". Nach (13) ist wieder $\lambda \leqq 1 /|1-\alpha|$. Es folgt aber wieder sogar (15). Denn es gibt eine entsprechende quasikonforme Abbildung der Vollebene auf sich, die $|\zeta-1|=1$ in $\mathfrak{Q}$ überführt, dabei innerhalb $|\zeta-1|<1$ konform ist. Eine solche Abbildung wird ja gegeben durch die Festsetzung $z=\zeta^{\alpha}$ für $\mathfrak{R e} z \equiv 0$ und $\log z=\log \zeta-$ $(1-\alpha)[\overline{\log \zeta}+i \pi]$ für $\mathfrak{R e} \zeta \leqq 0$. Für $\alpha=1 / 2$ erhalten wir insbesondere für eine Hälfte einer Lemniskate $\lambda=2$ (vgl. hierzu auch Ansätze in [3]), ebenso wie für den hieraus durch Stürzung entstehenden einen Ast einer gleichseitigen Hyperbel.

Die konstruierte quasikonforme Abbildung der Vollebene läßt noch eine große Willkür erkennen, so daß ebenfalls (15) gilt für eine große Klasse von Kurven $\mathfrak{L}$, die aus unserer , Knickkurve" durch Deformation innerhalb gewisser Grenzen entsteht. Dazu braucht in obiger Betrachtung die in der rechten Halbebene durch $z=\zeta^{\alpha}$ angesetzte Abbildung nur so abgeändert zu werden, daß die imaginäre Achse unverändert abgebildet wird, auch die abgeänderte Abbildung in $|\zeta-1|<1$ konform ist, aber im Rest der Halbebene z. B. für $\alpha<1$ noch [(2/ $\alpha)-1]$-quasikonform. Das abgeänderte $\mathfrak{L}$ sei also dabei das Bild von $|\zeta-1|=1$. Dies läuft nach Stürzung offenbar hinaus auf die Betrachtung der $[(2 / \alpha)-1]$-quasikonformen Abbildungen der rechten Halbebene in sich, bei denen die imaginäre Achse punktweis festbleibt und rechts einer gewissen Parallelen zur imaginären Achse Konformität vorliegt.

d) Ganz unmittelbar ergibt sich aus [8], Folgerung 2 (vgl. auch eine dort zitierte Arbeit von J. O. McLeavey) 
Satz 3. Ist $w(z) \in \Sigma$ und $z u$ einer stetigen schlichten Abbildung der $z$-Vollebene fortsetzbar, die $Q_{2}$-quasikonform ist in $|z|<R<1, Q_{1}$-quasikonform in $R<|z|<1$, dann gilt mit $q_{k}=\left(Q_{k}-1\right) /\left(Q_{k}+1\right)$

$$
w(z) \in \sum\left[\frac{q_{1}\left(1-q_{1} q_{2}\right)+\left(q_{2}-q_{1}\right) R^{2}}{1-q_{1} q_{2}+q_{1}\left(q_{2}-q_{1}\right) R^{2}}\right] \text { für } \quad Q_{2} \geqq Q_{1},
$$

insbesondere für $q_{1}=0$ speziell $w(z) \in \Sigma\left[q_{2} R^{2}\right]$.

Dies kann nach [8] natürlich noch allgemeiner formuliert werden und liefert indirekt wieder Eigenwertabschätzungen; Resultate verwandter Tendenz in [14] und dort zitierter Literatur. Für $Q_{2}<Q_{1}$ folgt nur $w(z) \in \Sigma\left[q_{1}\right]$ statt (16), und dies läßt sich nicht verschärfen.

\section{Die Eigenwerte der Niveaulinien der Greenschen Funktion}

Zu jedem $w(z) \in \Sigma$ entstehen als Bilder von $|z|=\varrho>1$ gewisse ,Niveaulinien" $\mathcal{I}(\varrho)$, deren Eigenwerte $\lambda=\lambda(\varrho)$ wir nun studieren wollen. Falls $|z|=1$ in eine Jordankurve $\mathfrak{Q}=\mathfrak{Q}(1)$ übergeht, wird diese mit in die Betrachtung einbezogen. Entwickeln wir zu $w(z) \in \Sigma$ gemäß (3), so gilt für $w(\varrho z) / \varrho \in \Sigma$ bei $\varrho>1$

$$
-\log \frac{[w(\varrho z) / \varrho]-[w(\varrho \zeta) / \varrho]}{z-\zeta}=\sum_{k, l=1}^{\infty} a_{k l} \varrho^{-k-l} z^{-k \zeta-l}
$$

Dies liefert unmittelbar den

Satz 4. Wenn $w(z) \in \Sigma[\varkappa]$ ist, dann gilt $w(\varrho z) / \varrho \in \Sigma\left[x / \varrho^{2}\right] \quad z u \quad \varrho=1$.

$\mathrm{Ob}$ die entsprechende Aussage für $\Sigma(\varkappa)$ gültig ist, ist unbekannt; vgl. im unter [2] zitierten Sammelbande die sogar schwächere Aufgabe 6.79 auf S. 560.

Aus Satz 4 fließt weiter nach Satz 1 in [10] die

Folgerung 1. Es gilt $\lambda\left(\varrho_{1}\right) / \varrho_{1}^{2} \geqq \lambda\left(\varrho_{2}\right) / \varrho_{2}^{2}$ für $\varrho_{1} \geqq \varrho_{2} \geqq 1$. Jedenfalls ist $\lambda(\varrho)$ eigentlich monoton steigend.

Dabei sei bei Zulassung von $\varrho_{2}=1$ das Bild von $|z|=1$ eine Jordankurve und hierbei in [10] die Bemerkung in Klammern am SchluB von S. 127 beachtet.

Die Aussage der Folgerung für $\varrho_{2}>1$ wird aufwendiger durch Variationsbetrachtungen schon in [12], S. 1209/10 hergeleitet.

Nach [15] ist $\lambda(\varrho)$ für $\varrho>1$ eine stetige Funktion. Wegen der Monotonie existiert $\lim \lambda(\varrho)$ für $\varrho \rightarrow 1$. Im Verein mit der in [16] bewiesenen Halbstetigkeit liefert dies

Folgerung 2. Die Funktion $\lambda(\varrho)$ ist für $\varrho \geqq 1$ stetig, falls $\mid z=1$ in eine Jordankurve iubergeht.

Nun kommen wir zum schon in der Einleitung angekündigten 
Satz 5. Es gilt $\Sigma\langle\varkappa\rangle \equiv \Sigma[\varkappa]$ für $\varkappa<1$. Das heißt: Für jedes $w(z) \in \Sigma$, das auf's Äußere einer Jordankurve mit Eigenwert $\lambda$ abbildet, ist die Grunsky-Norm $=1 / \lambda$.

Beweis. Entsprechend der Einleitung brauchen wir nur noch $\Sigma[x] \subseteq \Sigma\langle x\rangle$ zu zeigen. Sei $w(z) \in \Sigma[\chi]$. Dann ist nach Satz 4 sowie [10] (dort Satz 1) wegen der Randregularität bei $w(\varrho z) / \varrho$ sicher $1 / \lambda(\varrho) \leqq x / \varrho^{2}$, und nach Folgerung 2 ergibt sich - wie behauptet $-1 / \lambda \leqq \varkappa$.

Beiläufig ergibt sich damit: Da $\Sigma[\chi]$ zu festem $\varkappa$ kompakt ist, gilt dies auch für $\Sigma\langle x\rangle$.

Nach Folgerung 1 existiert $\lim \lambda(\varrho) / \varrho^{2}$ für $\varrho \rightarrow \infty$. Über diesen Grenzwert erhalten wir noch

Satz 6. Bei der Entwicklung (2) für $w(z) \in \Sigma[\varkappa]$ gilt für $\varrho>1$

$$
\left|a_{1}\right| \varrho \leqq \varrho^{2} / \lambda(\varrho) \leqq\left|a_{1}\right|+\frac{\chi}{\sqrt{\varrho^{2}-1}}+\frac{\varkappa}{\varrho^{2}-1},
$$

insbesondere

$$
\lim _{\varrho \rightarrow \infty} \varrho^{2} / \lambda(\varrho)=\left|a_{1}\right| .
$$

Beweis. Die linke Seite von (18) ergibt sich aus (4) mit $n=1$, angewandt auf $w(\varrho z) / \varrho$ gemäß (17). Zur Herleitung der rechten Seite von (18) gehen wir aus von dem Ausdruck der linken Seite der Grunskyschen Bedingungen für $w(\varrho z) / \varrho$ und benutzen $\left|a_{k l}\right| \leqq x / \sqrt{k l}$ :

$$
\begin{gathered}
\left|\sum_{k, l=1}^{n} a_{k l} \varrho^{-k} x_{k} \varrho^{-l} x_{l}\right| \\
=\left|a_{11} \varrho^{-2} x_{1}^{2}+\sum_{k, l=2}^{n} a_{k l} \varrho^{-k} x_{k} \varrho^{-l} x_{l}+2 \sum_{k=2}^{n} a_{k 1} \varrho^{-k} x_{k} \varrho^{-1} x_{1}\right| \\
\leqq\left|a_{11}\right| \varrho^{-2}\left|x_{1}\right|^{2}+x\left(\sum_{k=2}^{n} \varrho^{-k}\left|x_{k}\right| / \sqrt{k}\right)^{2}+2 \varkappa \varrho^{-1}\left|x_{1}\right| \sum_{k=2}^{n} \varrho^{-k}\left|x_{k}\right| / \sqrt{k} \\
\leqq\left\{\left|a_{11}\right| \varrho^{-2}+\frac{\varrho^{-2} \varkappa}{\varrho^{2}-1}+\frac{\varrho^{-2} x}{\sqrt{\varrho^{2}-1}}\right\} \sum_{k=1}^{n}\left|x_{k}\right|^{2} / k .
\end{gathered}
$$

was wir haben wollten.

Dabei benutzen wir $u$. a. noch die Schwarzsche Ungleichung

$$
\begin{gathered}
\left(\sum_{k=2}^{n} \varrho^{-k}\left|x_{k}\right| / \sqrt{k}\right)^{2} \leqq \sum_{k=2}^{n} \varrho^{-2 k} \cdot \sum_{k=2}^{n}\left|x_{k}\right|^{2} / k \leqq \frac{Q^{-2}}{Q^{2}-1} \sum_{k=2}^{n}\left|x_{k}\right|^{2} / k, \\
2\left|x_{1}\right|\left(\sum_{k=2}^{n}\left|x_{k}\right|^{2} / k\right)^{1 / 2} \leqq\left|x_{1}\right|^{2}+\sum_{k=2}^{n}\left|x_{k}\right|^{2} / k .
\end{gathered}
$$

Falls $a_{1}=0$, kann man statt Satz 6 eine entsprechende schärfere Aussage über das Abklingen von $1 / \lambda(\varrho)$ für $\varrho \rightarrow \infty$ bekommen. Ist nämlich $a_{1}=0$, so erhält man 
ähnlich wie oben

$$
\sqrt{2}\left|a_{2}\right| \leqq \varrho^{3} / \lambda(\varrho) \leqq \frac{x}{\sqrt{1-\varrho^{-2}}}+\frac{x / \varrho}{1-\varrho^{-2}},
$$

und dies kann man wiederum verschärfen, wenn noch $a_{2}=0$ ist, usw.

Es bleiben bei der Funktion $\lambda(\varrho)$ noch viele Fragen offen, z.B. die nach der Existenz von $\lambda^{\prime}(\varrho)$ oder gar die, ob $\lambda(\varrho)$ reell-analytisch ist.

\section{Zusatzbemerkungen}

a) Es ist nicht nur $\Sigma(\varkappa) \neq \Sigma[\varkappa]$ (vgl. Einleitung), sondern man kann auch explizit ein einfaches Beispiel eines Extremalproblems angeben, das in $\Sigma(x)$ und $\Sigma[x]$ verschiedene Lösungen hat. Das Extremalproblem $\left|a_{2}\right| \rightarrow \max$ (vgl. (2)) wird nämlich nach [7] durch die scharfe Ungleichung $\left|a_{2}\right| \leqq(2 / 3) x$ in $\Sigma(x)$ gelöst. Diese Ungleichung kann jedoch nicht in $\Sigma[x]$ bzw. $\Sigma\langle x\rangle$ gültig sein, da die in [7] unter (17) (dort mit $k=2, q=x)$ angegebenen Funktionen nach [10], $\S 3 \mathrm{zu} \Sigma\left[x^{\prime}\right]$ mit jeweils einem gewissen $x^{\prime}<\varkappa$ gehören. Insbesondere kann also die Ungleichung $\left|a_{2}\right| \leqq(2 / 3) \varkappa$ für die Klasse $\Sigma(x)$ gar nicht allein mit den verallgemeinerten Grunskyschen Koeffizientenbedingungen (4) beweisbar sein - vgl. hierzu [7] unter IV. Nach dem bekannten aus (4) folgenden verallgemeinerten Flächensatz gilt in $\Sigma[\varkappa]$ bekanntlich $\Sigma n\left|a_{n}\right|^{2} \leqq \chi^{2}$, also immerhin $\left|a_{2}\right| \leqq \varkappa / \sqrt{2}$, was freilich nicht scharf sein kann. Es wäre wünschenswert, die Theorie der Extremalprobleme in $\Sigma[\varkappa]$ nach Variationsmethoden auszubauen, etwa den Ansätzen in [14] (dort weitere Literatur) folgend; auch das Studium von Symmetrisierungsprozessen wäre interessant.

Obige Betrachtung bringt indirekt auch eine Eigenwertabschätzung für die in [10], $\S 3$ betrachtete dreifach symmetrische Kurve $\mathcal{Q}$. Für die dort unter (11) angegebene Abbildung $w(z)$ ist nämlich $\left|a_{2}\right|=(2 / 3) r^{2}$, und dies muß nach dem eben Angegebenen wegen $w(z) \in \Sigma[1 / \lambda]$ also $<(1 / \lambda) / \sqrt{2}$ sein. Dies bringt

$$
1>\frac{1 / \lambda}{r^{2}}>\frac{2 \sqrt{2}}{3}=0,9428 \ldots
$$

wobei $1 / \lambda<r^{2}$ nach [10] gilt. Die Bestimmung des genauen Wertes von $\lambda$ bleibt ein offenes Problem.

b) Im Anschluß an die Feststellung $\Sigma(\varkappa) \neq \Sigma[\varkappa]$ entsteht die Frage, unter welchen zusätzlichen Voraussetzungen eine Abbildung $w(z) \in \Sigma[\varkappa]$ auch $z u \quad \Sigma(x)$ gehört. Eine entscheidende Rolle spielen dabei wohl Eigenschaften der zum betreffenden Bild $\mathfrak{L}$ von $|z|=1$ gehörenden Eigenfunktionen. Wir wollen uns im folgenden auf analytische $\mathfrak{Q}$ beschränken und mit $F(w)$ die im Anschluß an Schiffer in [9] betrachteten komplexen Eigenfunktionen bezeichnen. Das sind außerhalb $\mathfrak{Q}$ reguläre Funktionen $\neq \equiv 0$, die in $w=\infty$ verschwinden, für die $F-\mu \bar{F}$ ins Innere von $\mathfrak{I}$ 
analytisch fortsetzbar ist, wobei auf $\mathcal{Q}$ noch Stetigkeit und damit Regularität vorliegt. Jetzt bezeichne $\mu>1$ irgendeinen Fredholmschen Eigenwert (also nicht nur den kleinsten). Es gilt folgende (allerdings nur hinreichende) Bedingung.

Satz 7. Wenn eine komplexe Eigenfunktion $F(w)$ mit Eigenwert $\mu=1$ außerhalb von $\mathbb{Z}$ und innerhalb von $\mathfrak{\&}$ jeweils schlicht ist, besitzt das zugehörige $w(z)$ nach $|z|<1$ eine quasikonforme Fortsetzung mit dem konstanten Betrag $1 / \mu$ der komplexen Dilatation, und diese Abbildung ist bezüglich der auf $|z|=1$ gegebenen Randabbildung die eindeutig bestimmte möglichst konforme (=extremal quasikonforme) Abbildung. Insbesondere kann immer höchstens zu einem Eigenwert $\mu$ von $\mathfrak{I}$, nämlich dem kleinsten $=\lambda$, die besagte Schlichtheit bei $F(w)$ auftreten. Jedenfalls folgt dann aus $w(z) \in \Sigma[\chi]$ auch $w(z) \in \Sigma(\chi)$, falls zum Bild ron $|z|=1$ die besagte Schlichtheit bei einer zugehörigen komplexen Eigenfunktion vorliegt.

Beweis. Diese quasikonforme Fortsetzung nach $|z|<1$, sagen wir $w^{*}(z)$, wird so geliefert. Zu $z$ mit $|z|<1$ suche man denjenigen eindeutig (wegen der Schlichtheit von $F(w)$ innerhalb $\mathfrak{Q})$ bestimmten Wert $w^{*}$ innerhalb $\mathbb{2}$ mit $F\left(w^{*}\right)=F(w(1 / z))$. Die Funktion $w^{*}(z)$ ist offenbar bezüglich der auf $|z|=1$ vorliegenden Randwerte ,teichmüllersch", so daß die Extremalität z. B. nach [19] folgt. Der Rest der Behauptung folgt aus $1 / \lambda \leqq \%$, wenn zu $w(z)$ eine quasikonforme Fortsetzung mit der Schranke $\varkappa$ für den Betrag der komplexen Dilatation existiert [1].

Als Anwendung diese Satzes ergibt sich, daß zu der schon oben am Schluß von Zusatzbemerkung a) genannten dreifach symmetrischen Kurve für keine einzige komplexe Eigenfunktion $F(w)$ die im Satz 7 genannte Schlichtheit vorliegen kann.

Man kann übrigens auch die Schlußweise im Beweis von Satz 7 umkehren und aus Eigenschaften einer möglichst konformen quasikonformen Fortsetzung nach $|z|<1$ (nämlich im wesentlichen der Eigenschaft, teichmüllersch zu sein ohne Nullstellen des zugehörigen quadratischen Differentials) auf eine außerhalb und innerhalb vom zugehörigen $\mathfrak{Q}$ jeweils schlichte komplexe Eigenfunktion schließen.

\section{Literatu:}

[1] Ahlfoks, L. V.: Remarks on the Neumann-Poincaré integral equation. - Pacific J. Math. 2, 1952. $271-280$.

[2] BeCKer, J.: Conformal mappings with quasiconformal extensions. - Aspects of Contemporary Complex Analysis, Proc. Conf. Durham, 1979, Academic Press, LondonNew York-Toronto-Sydney-San Francisco, 1980, 37-77.

[3] Blumenfeld, J., und W. Mayer: Über Poincaré'sche Fundamentalfunktionen. - Sitzungsber. Math.-Natur. KI. Kaiserl. Akad. Wiss. Wien 123 (Abt. IIa), 1914, 2011 -2047.

[4] KüHNAu, R.: Verzerrungssätze und Koeffizientenbendingungen vom Grunskyschen Typ für quasikonforme Abbildungen. - Math. Nachr. 48, 1971, 77-105.

[5] KüHNAU, R.: Eine funktionentheoretische Randwertaufgabe in der Theorie der quasikonformen Abbildungen. - Indiana Univ. Math. J. 21, 1971, 1-10. 
[6] Kühnau, R.: Weitere elementare Bemerkungen zur Theorie der konformen und quasikonformen Abbildungen. - Math. Nachr. 51, 1971, 377-382.

[7] KüHNAU, R.: Zum Koeffizientenproblem bei den quasikonform fortsetzbaren schlichten konformen Abbildungen. - Math. Nachr. 55, 1973, 225-231.

[8] KüHNAU, R.: Extremalprobleme bei quasikonformen Abbildungen mit kreisringweise konstanter Dilatationsbeschränkung. - Math. Nachr. 66, 1975, 269-282.

[9] KüHNAU, R.: Eine Integralgleichung in der Theorie der quasikonformen Abbildungen. Math. Nachr. 76, 1977, 139-152.

[10] Kühnau, R.: Zu den Grunskyschen Koeffizientenbedingungen. - Ann. Acad. Sci. Fenn. Ser. A I Math. 6, 1981, 125-130.

[11] Pommerenke, Chr.: Univalent functions. - Verlag Vandenhoeck \& Ruprecht, Göttingen, 1975.

[12] Schiffer, M.: The Fredholm eigenvalues of plane domains. - Pacific J. Math. 7, 1957 , $1187-1225$.

[13] Schiffer, M.: Fredholm eigenvalues and Grunsky matrices. - Ann. Polon, Math. 39, 1981, $149-164$.

[14] Schiffer, M., and G. Schober: An extremal problem for the Fredholm eigenvalues. - Arch. Rational Mech. Anal. 44, 1971, 83-92.

[15] Schober, G.: Continuity of curve functionals and a technique involving quasiconformal mapping. - Arch. Rational Mech. Anal. 29, 1968, 378-389.

[16] Schober, G.: Semicontinuity of curve functionals. Arch. Rational Mech. Anal. 33, 1969, $374-376$.

[17] Schober, G.: Estimates for Fredholm eigenvalues based on quasiconformal mapping. Lecture Notes in Mathematics 333, Springer-Verlag, Berlin-Heidelberg-New York, 1973, 211-217.

[18] Springer, G.: Fredholm eigenvalues and quasiconformal mapping. - Acta Math. 111, 1964, $121-142$.

[19] Strebel, K.: Zur Frage der Eindeutigkeit extremaler quasikonformer Abbildungen des Einheitskreises. - Comment. Math. Helv. 36, 1962, 306-323.

20] Tsusi, M.: Potential theory in modern function theory. - Maruzen Co., Ltd., Tokyo, 1959.

\author{
Martin-Luther-Universität Halle-Wittenberg \\ Section Mathematik \\ DDR - 401 Halle an der Saale \\ Deutsche Demokratische Republik
}

Eingegangen am 11. Juni 1982. 